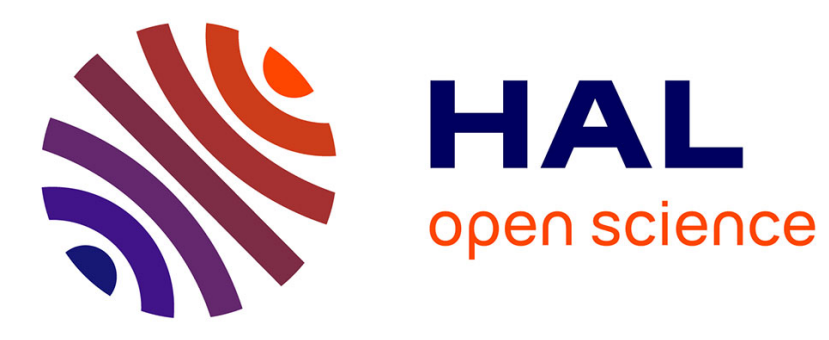

\title{
A Geant4 simulation for three-dimensional proton imaging of microscopic samples
}

Claire Michelet, Zhuxin Li, Wen Yang, Sébastien Incerti, Pascal Desbarats, Jean-François Giovannelli, Philippe Barberet, Marie-Hélène Delville, Nuria Gordillo, Guillaume Devès, et al.

\section{To cite this version:}

Claire Michelet, Zhuxin Li, Wen Yang, Sébastien Incerti, Pascal Desbarats, et al.. A Geant4 simulation for three-dimensional proton imaging of microscopic samples. Physica Medica, 2019, 65, pp.172-180. 10.1016/j.ejmp.2019.08.022 . hal-02281684

\section{HAL Id: hal-02281684 https://hal.science/hal-02281684}

Submitted on 4 Nov 2019

HAL is a multi-disciplinary open access archive for the deposit and dissemination of scientific research documents, whether they are published or not. The documents may come from teaching and research institutions in France or abroad, or from public or private research centers.
L'archive ouverte pluridisciplinaire HAL, est destinée au dépôt et à la diffusion de documents scientifiques de niveau recherche, publiés ou non, émanant des établissements d'enseignement et de recherche français ou étrangers, des laboratoires publics ou privés. 
Original article: Physica Medica 65 (2019) 172-180

DOI : https://doi.org/10.1016/j.ejmp.2019.08.022

\section{A Geant4 simulation for three-dimensional proton imaging of microscopic samples}

Claire Michelet ${ }^{\mathrm{a}}$, Zhuxin $\mathrm{Li}^{\mathrm{a}}$, Wen Yang ${ }^{\mathrm{a}}$, Sébastien Incertia ${ }^{\mathrm{a}}$, Pascal Desbarats ${ }^{\mathrm{b}}$, Jean-François Giovannellic $^{\mathrm{c}}$, Philippe Barberet ${ }^{\mathrm{a}}$, Marie-Hélène Delville ${ }^{\mathrm{d}}$, Nuria Gordillo ${ }^{\mathrm{a}, 1}$, Guillaume Devès ${ }^{\mathrm{a}}$, Hervé Seznec ${ }^{\mathrm{a}}$

${ }^{a}$ CENBG, UMR5797, Université de Bordeaux, CNRS, F-33170, Gradignan, France

${ }^{b}$ Laboratoire Bordelais de Recherche en Informatique (LaBRI, UMR5800) Univ. Bordeaux, 351 Cours de la Libération, F-33405 Talence cedex, France

${ }^{c}$ IMS (Univ. Bordeaux, CNRS, BINP), 351 Cours de la Libération, F-33405 Talence, France

d CNRS, Univ. Bordeaux, ICMCB, UMR5026, 87 avenue du Dr. A. Schweitzer, Pessac, F33608, France

\section{E-mail addresses:}

Claire MICHELET michelet@ cenbg.in2p3.fr

Zhuxin LI zhuxin.li@outlook.com

Wen YANG yangwen223@outlook.com

Sébastien INCERTI incerti@cenbg.in2p3.fr

Pascal DESBARATS pascal.desbarats@labri.fr

Jean-François GIOVANNELLI giova@ims-bordeaux.fr

Philippe BARBERET barberet@ cenbg.in2p3.fr

Marie-Hélène DELVILLE marie-helene.delville@icmcb.cnrs.fr

Nuria GORDILLO nuri.gordillo@gmail.com

Guillaume DEVES deves@cenbg.in2p3.fr

Hervé SEZNEC herve.seznec@cenbg.in2p3.fr

${ }^{1}$ Present address, Nuria Gordillo:

Grupo de Electrónica y Semiconductores, Departamento de Física Aplicada, Universidad Autónoma de Madrid, Cantoblanco, E-28049 Madrid, Spain

*Corresponding author.

E-mail address: michelet@cenbg.in2p3.fr (C. Michelet).

Keywords:

Geant4 simulation

STIM tomography

PIXE tomography

Caenorhabditis elegans 


\begin{abstract}
Proton imaging can be carried out on microscopic samples by focusing the beam to a diameter ranging from a few micrometers down to a few tens of nanometers, depending on the required beam intensity and spatial resolution. Three-dimensional (3D) imaging by tomography is obtained from proton transmission (STIM: Scanning Transmission Ion Microscopy) and/or Xray emission (PIXE: Particle Induced X-ray Emission). In these experiments, the samples are dehydrated for under vacuum analysis. In situ quantification of nanoparticles has been carried out at CENBG in the frame of nanotoxicology studies, on cells and small organisms used as biological models, especially on Caenorhabditis elegans (C. elegans) nematodes. Tomography experiments reveal the distribution of mass density and chemical content (in g. $\mathrm{cm}^{-3}$ ) within the analyzed volume. These density values are obtained using an inversion algorithm. To investigate the effect of this data reduction process, we defined different numerical phantoms, including a (dehydrated) $C$. elegans phantom whose geometry and density were derived from experimental data. A Monte Carlo simulation based on the Geant4 toolkit was developed. Using different simulation and reconstruction conditions, we compared the resulting tomographic images to the initial numerical reference phantom. A study of the relative error between the reconstructed and the reference images lead to the result that 20 protons per shot can be considered as an optimal number for 3D STIM imaging. Preliminary results for PIXE tomography are also presented, showing the interest of such numerical phantoms to produce reference data for future studies on X-ray signal attenuation in thick samples.
\end{abstract}

\title{
1. Introduction
}

The technique of proton computed tomography (pCT) finds its origin in the first experiments carried out in the seventies by Cormack and Koehler [1] with a $158 \mathrm{MeV}$ proton beam, $2 \mathrm{~mm}$ in diameter, on lucite phantoms a few centimeters in size. It was shown that a better density contrast was achieved with protons compared to X-rays, for a lesser deposited dose. For microscopic samples, pCT was developed using lower energy (a few MeV) accelerators. A review was published [2], giving historical aspects as well as technical details about experimental conditions and data reduction. To summarize the experimental procedure, the proton beam is focused using magnetic quadrupoles, down to a diameter from a few tens of nanometers up to a few micrometers, depending on the performances of the beam line and on the required beam intensity and/or spatial resolution. The beam is scanned over the region of interest (ROI) of the sample. Two tomography techniques are carried out: transmission tomography (STIM) and X-ray emission tomography (PIXE). In its principle, STIM is similar to pCT: the remaining energy of the protons after going through the sample is measured, at each position of the beam. PIXE consists in collecting the X-rays emitted by the atoms of the sample, following their ionization by the incident protons, at each position of the beam. This gives access to the spatial distribution of all the chemical elements (for $Z \geq 11$, using conventional thin window X-ray detectors), in a quantitative way. The sensitivity of the technique (down to a few $\mu \mathrm{g} \cdot \mathrm{g}^{-1}$ in concentration) is a major advantage. On-going developments of STIM and PIXE tomography address:

- Increasing data statistics and/or reducing beam time for PIXE, by increasing the detection solid angle, using two X-ray detectors at the same time [3, 4].

- Correcting data for X-ray signal attenuation into the sample [3-7]. This attenuation is caused, first, by the variation of ionization cross section, which decreases due to the proton energy decrease into the sample, resulting in a decrease in X-ray production. A 
second issue is X-ray absorption, which occurs into the sample, along the path from the emission points to the detector.

An attempt to evaluate the accuracy of the reconstruction process for STIM and PIXE tomography was performed by comparing the results obtained from the same experimental data set, using different data reduction and tomographic reconstruction methods $[3,5]$ compared to one another. A precise study would require using numerical phantoms of defined density and composition, in order to compare the reconstructed images with the original phantoms. The purpose of the present work is to answer this issue, by developing a Geant4 (http://geant4.org) [8-10] based simulation code to model STIM and PIXE tomography and by designing numerical phantoms to serve as a reference to evaluate the effect of the reconstruction process and its ability to calculate accurate values when compared to the original reference densities defined in the phantom. More precisely, our purpose was to answer the following questions: Is the shape of the phantom preserved by the tomographic reconstruction? What is the accuracy of the reconstructed values? What is the influence of the reconstruction method? What is the influence of the number of protons used? And more particularly: what minimal number of protons is required? To answer these questions, the reconstructed images were analyzed at the global and local levels (as explained in section 2.8), using different number of protons for the simulation and comparing two different tomographic reconstruction methods usually implemented for STIM and PIXE tomography.

In this study, we present for the first time Geant4 phantoms and simulation codes designed for STIM and PIXE tomography at microscopic level, with proton beams in the range of a few $\mathrm{MeV}$. We took into account the guidelines for publication of Monte Carlo studies formulated by Sechopoulos et al. [11]. To broaden the context of this work, it should be mentioned that the Geant4 toolkit has been widely used in the field of macroscopic $p C T$, either directly or using specific Geant4-based tools suited to medical imaging and radiotherapy, such as GATE (http://www.opengatecollaboration.org) or TOPAS (http://www.topasmc.org). Some of the main topics address the evaluation of the accuracy when determining: ( $i$ ) dose maps and proton ranges, compared to conventional X-ray scanners [12, 13], (ii) relative stopping power, using high spatial resolution voxelized phantoms [14], (iii) individual proton tracking, with the purpose to optimize the experimental setup [15-16] and/or the reconstruction $[17,18]$.

\section{Material and methods}

\subsection{Definition of the general set up and selection of the physics models}

The simulation of PIXE spectra had been already implemented in Geant4 by Incerti et al. [19] for a fixed 1.5 MeV proton beam, on thin reference samples homogeneous in thickness and composition. A good agreement was obtained with experimental spectra. Following the recommendations of this study, we started from the TestEm5 code, taken from the set of Geant4 (2017-10.3 version) "extended" examples, allowing in particular the simulation of electromagnetic interactions at low energy. As suggested by Incerti et al. [19] the low energy G4EmLivermorePhysics electromagnetic physics class (abbreviated as "Livermore" model) was implemented [20], as it takes into account atomic shells and is well suited for the simulation of X-ray emission processes. For the simulation of X-ray fluorescence lines, the set of Geant4 files from the Bearden database [21] has been used for a better accuracy on the energy of emitted X-rays. To reduce the duration of the simulation, the energy cutoff for the simulation of secondary particles was set to $990 \mathrm{eV}$ - knowing that the "Livermore" database can actually be used down to a few tens of $\mathrm{eV}$ (with reduced accuracy). We should note that the $990 \mathrm{eV}$ energy limit only deals with the production of secondary particles and that the X- 
rays we are interested in are above this limit. It was checked that, as expected, this energy limitation did not affect the production of X-rays of interest.

Two Geant 4 methods were used to get the energy of the transmitted protons and of the X-rays going out of the object: (i) at the step simulation level, more precisely when protons are going out of the phantom; (ii) at the end of the track (as the phantom is surrounded by vacuum). Both should obviously give the same results. This comparison was used to check the consistency of the simulation results. Anomalous significant differences between the two methods were found until a proper limitation was set on the maximal step length. Because the phantom volumes are small (a few tens of micrometers at most) and of low density, some particles could go through without a step point being generated. The step limitation was set such as at least 10 steps were performed in each traversed volume. After these tests were completed, we finally collected transmitted proton and X-ray data at the end of the track.

\subsection{Numerical phantoms}

Two types of numerical phantoms were designed. The first one was a homogeneous cube of user-defined size, density and composition. The second one was a more realistic phantom of a dehydrated biological sample, a $C$. elegans worm. C. elegans is a transparent nematode about 1 millimeter in length living in most terrestrial ecosystems and widely used as a model in Biology (http://www.wormbook.org/). At CENBG, C. elegans worms are cultivated to study the effect of the presence of titanium dioxide $\left(\mathrm{TiO}_{2}\right)$ nanoparticles in the growth medium [22]. Sample cryofixation followed by freeze-drying is required for the ion beam analysis of biological materials as the beamline and analysis chamber are under vacuum [23, 24]. Specific protocols have been designed, in order to preserve the sample structure and elemental composition at the cell level [25]. The precise localization and quantification of nanoparticles within C. elegans has motivated the use of 3D tomography techniques [26, 27].

The upper part of such a dehydrated $C$. elegans worm was modeled using six ellipsoids to represent six specific regions (Fig. 1): the cuticle ("skin" of the worm), the body, two cell nuclei (cells forming the intestine), the intestine and a Ti rich region inside the intestine. For this study, we selected a ROI at the beginning of the intestine, where $\mathrm{TiO}_{2}$ nanoparticles were found experimentally. A slice of interest was chosen through the cell nuclei and the Ti rich region. The shape, size, density and element content of the phantom were obtained from experimental data from freeze dried specimens [26, 27]. The main features of data reduction will be detailed thereafter, when describing the reconstruction process with the Geant4 simulated data. The phantom was designed according to the values in Tables 1 and 2, measured from the experimental tomographic images reconstructed using a filtered back projection algorithm. For this, in the Geant 4 simulation, we defined the individual chemical elements using the G4Element class, which takes into account natural isotopic abundances. The materials constituting the different regions of the C. elegans phantom were defined using the G4Material class, by adding the elements according to their corresponding mass fractions and specifying the total mass density in the region (Table 2).

Table 1 was obtained from STIM tomography, as this technique gives the 3D structure by density contrast. More precisely, by calculating the sample's stopping power (assuming a global homogeneous composition for the major elements), quantitative STIM tomography imaging gives access to the 3D distribution of mass density, expressed in $\mathrm{g}_{\mathrm{cm}} \mathrm{cm}^{-3}$. Table 2 displays the composition of the different regions of the phantom. To our knowledge, the complete composition of dehydrated $C$. elegans has not yet been determined experimentally. We should remind that our purpose in the present study is not to determine the exact real 
composition of a $C$. elegans, but to study the effect of the data processing algorithm producing the tomographic images on the accuracy of the density determination. For this, our reference is the Geant4 numerical phantom. We tried to make this phantom as realistic as possible using the experimental data available to date, however its composition cannot be considered as an accurate model of a real C. elegans.

We estimated the composition of the $C$. elegans phantom from experimental data available at CENBG. The element content for $\mathrm{P}, \mathrm{S}, \mathrm{Cl}, \mathrm{K}$ and $\mathrm{Ti}$ was obtained from experimental PIXE tomography images (one image per chemical element) displaying the element mass density distribution (in ${\mathrm{g} . \mathrm{cm}^{-3}}^{-3}$ ). These values were then converted to mass percentage, as required to define the composition of Geant4 materials (Table 2). The rest of the material was considered as (dry) biological material. Its composition $\mathrm{C}_{10} \mathrm{H}_{17} \mathrm{O}_{3} \mathrm{~N}_{2}$ was derived from previous studies of cryofixed and lyophilized biological samples by RBS (Rutherford Backscattering Spectrometry) using classical (not tomographic) 2D ion beam analysis [28]. RBS spectra give access to the composition of the major elements present in organic materials, more precisely to carbon, nitrogen, oxygen. It is usually considered that the amount of hydrogen cannot be determined from RBS. Indeed, from a kinematics point of view, when using a proton beam, there cannot be any backscattering peak for hydrogen (the lightest element that could appear would be helium). However, for biological samples, the amount of hydrogen can be indirectly determined from RBS spectra considering its influence on the height and width of the peaks of the other elements. Such a determination is not straightforward and requires a specific experimental protocol: a thin foil of certified thickness and composition is placed on the sample, so that it partly recovers the area of interest. This foil serves as a reference for the quantitative analysis of RBS spectra. In this way, the amount of hydrogen can be determined [29]. Such experiments have not yet been performed for C. elegans. So in this study, the composition $\mathrm{C}_{10} \mathrm{H}_{17} \mathrm{O}_{3} \mathrm{~N}_{2}$ used for the biological material remains arbitrary. Nevertheless, as discussed in section 2.6, the precise knowledge of this composition does not have a high impact on the final calculation of sample thickness.

\subsection{Definition of the scan}

Just as for real 3D tomography experiments, in the Geant 4 simulation the beam is scanned over the area of interest following a $2 \mathrm{D}$ grid (Fig. 2). The scan width and scan height are defined by the user, as well as the position of the scan in the vertical $(z)$ direction, if only a specific part of the phantom should be analyzed. At each position of the source, the energy of the protons (for STIM) and/or the emitted X-rays (for PIXE) going out of the sample is recorded. At the first projection $\left(0^{\circ}\right.$ angle), the proton beam is defined to follow the $x$ axis. Once the whole ROI has been scanned at this angle, the grid modeling the different positions of the source is rotated - and the direction of the beam is rotated accordingly - to perform the next projection. The results of 3D STIM tomography presented hereafter for the $C$. elegans phantom were obtained using a $128 \times 128$ scan grid, typical of real experiments, resulting in 128 slices of $128 \times 128$ pixels. The angular step was $1.8^{\circ}$, corresponding to 100 projections taken over the $\left[0^{\circ} ; 180^{\circ}\right.$ [ angle range. The numerical superposition of the reconstructed slices gives the final 3D image.

A macro file drives the source parameters: energy of incident protons, number of protons per shot, definition of the scan grid. The source is controlled using the General Particle Source (GPS) primary particle generator. The successive positions and directions of the source are generated automatically by a Python script, according to the user's specifications. As for real experiments, the simulations presented here were carried out using a $1.5 \mathrm{MeV}$ proton beam. 


\subsection{Recording simulated data}

Proton and X-ray energies are recorded as ntuples of the ROOT data analysis toolkit (https://root.cern.ch/). Considering the primary protons at the end of the track, their angular deviation relative to the incident direction is calculated and also recorded. Only the protons having a small diffusion angle (user-defined value, $\pm 7^{\circ}$ in the simulations presented here) are kept for further reconstruction, so that they do not alter the calculation of the transmitted energy. Nevertheless, we should note that, for these phantoms, very few incident protons are discarded this way, as the size of the samples is small: for instance only $\sim 1 \%$ when the beam is going through the whole diameter of the C. elegans phantom [Fig. S1 in Supplementary material]. For this particular sample, we can conclude that the angle limitation is not useful, as the energy spectrum would be almost unchanged if we consider all the transmitted protons. Moreover, even if no angular limitation was considered, the median filtering process applied on transmitted energy values would in any case discard spurious events (as explained in section 2.6). To give a comparison with real experimental conditions, the experimental angle limitation due to the detector aperture is about $25^{\circ}$, considering that the protons are collected in a passivated implanted planar silicon detector (Canberra PIPS detector, $25 \mathrm{~mm}^{2}, 12 \mathrm{keV}$ energy resolution), placed at $0^{\circ}$ on the incoming beam axis, about $6 \mathrm{~mm}$ away from the sample.

For PIXE, each chemical element is identified from the energy of the X-ray lines in the energy spectrum (Fig. S2 in Supplementary material). The X-ray detector is placed at a given (user-defined) angle and with a given angular aperture. To avoid re-doing the simulation every time we change these parameters, a selection of the X-rays according to their direction is performed post-simulation using a $\mathrm{C}$ script. For this, the position of the emitted X-ray is recorded at the end of the track, meaning when arriving at the limit of the entire simulation volume, which is defined as a large sphere whose radius is about 20 times larger than the phantom size. The X-ray track is kept for further processing only if its direction intercepts the disk representing the user-defined detector.

\subsection{Execution}

The simulations were executed on three different setups, according to the expected duration:

- Monothreading (1 core, $3 \mathrm{GHz}, 24 \mathrm{~Gb}$ RAM) using a free access VMWare ${ }^{\circledR}$ (https://www.vmware.com/) virtual environment designed at CENBG (http://www.cenbg.in2p3.fr/-VMWare-et-Geant4-)

- Parallel computing (cluster with 80 cores, $2.33 \mathrm{GHz}, 16$ Gb RAM per core)

- Multithreading (28 threads on a multi-core computer $2.2 \mathrm{GHz}, 32 \mathrm{~Gb}$ RAM).

Particular attention was paid to properly set the random seed for parallel or multithreading computing and to check the consistency of the simulated data between the three configurations. For 3D STIM, the duration for 64 slices of the cubic phantom with 100 protons per shot was 5.5 hours on 1 core. For the $C$. elegans phantom, the maximal step length limitation increases computing time: it takes 2 days on 1 core, 128 slices, with 20 protons per shot. To compare with real experimental conditions, the typical duration of a 3D STIM tomography experiment is about 2.5 hours, for a number of protons per position of the beam usually between 10 and 20 (it may be more, according to the experimental conditions).

For PIXE tomography, a major concern is the long duration of simulations, due to the relatively low X-ray production cross sections and low concentrations of mineral and metal 
elements in biological samples. Experimental conditions at CENBG for such samples require about $10^{8}$ to $10^{9}$ protons per position of the beam, using a $\mathrm{Si}(\mathrm{Li})$ spectrometer (e2v Sirius detector, $80 \mathrm{~mm}^{2}, 148 \mathrm{eV}$ resolution for the $\mathrm{Mn} \mathrm{K}_{\alpha}$ line) placed at $22 \mathrm{~mm}$ of the sample, which corresponds to a detection aperture angle of about $\pm 13^{\circ}$ [27]. Keeping such conditions for the simulations would lead to too long computation times for the C. elegans phantom. To overcome this difficulty, we present here a preliminary study of PIXE tomography with modified conditions, in order to achieve simulations in a reasonable time. The results presented here were obtained using a larger aperture angle $\left( \pm 60^{\circ}\right)$. Moreover, we artificially increased the element content in the $C$. elegans phantom. The mass density of $\mathrm{P}, \mathrm{S}, \mathrm{Cl}, \mathrm{K}, \mathrm{Ca}$ was multiplied by a factor of 10 and the Ti content by a factor of 1000 compared to the original values reported in Table 2 . In this way, $10^{6}$ incident protons per shot were sufficient. In these conditions, for one PIXE slice, it took 8 days for the $40 \mu \mathrm{m}$ cubic phantom using 28 threads and 19 days for the (modified) C. elegans phantom (also about $40 \mu \mathrm{m}$ in width). To give a comparison with experimental conditions: it takes about 2 hours to collect data for one PIXE slice. A solution to overcome this problem of computing time for PIXE tomography simulation in the future could be to develop this application using a GPU architecture. This type of development has recently been carried out in the frame of the Geant4-DNA project [30]. It keeps the accuracy of the Geant 4 simulations whilst speeding up simulations with impressive performance gain (about 250 times faster).

\subsection{Calculation of tomographic sinograms}

After completing the simulation, the ntuples are rewritten into a binary format file for subsequent tomographic reconstruction, containing the energy, the position indices of the source in the scan grid and the projection number. These data are processed to obtain the tomographic sinograms. For each slice, the sinogram is the set of projected data corresponding to the value of the physical quantity of interest at each position of the beam and for each projection angle. For STIM, this physical quantity is the proton energy loss. For PIXE, it is the number of detected X-rays for each chemical element. The main steps of data reduction have been already detailed in previous publications [26, 27]. A critical point for proton transmission tomography is to evaluate the most probable value of the energy distribution at each position of the beam. It can be obtained from a Gaussian fit of the energy spectrum, for (microscopic) STIM tomography, or of the high-energy part of this spectrum, for macroscopic pCT. Actually, STIM tomography data reduction does not proceed by fitting this Gaussian shape. Pioneering studies on STIM image formation explored three different techniques to extract images from proton energy spectra (taken at each position of the beam): (i) summing of events selected within a specific energy window, (ii) energy averaging and (iii) median filtering of energy values [31]. It was demonstrated that median filtering was well suited to reduce noise and to sharpen boundaries. Indeed, it determines efficiently a good estimate of the most probable energy value from the distribution, without being affected by spurious events, which can be for instance, considering real experimental data: diffusion at low energy, pile up, non-resolved structures within the beam track, partial volume effects on the edges. Since then, median filtering is usually preferred for its robustness for "classical" 2D STIM and also for STIM tomography, especially in the case of low statistics and/or noisy data. For this reason, median filtering was used here to process the tomographic data and select the value of the proton transmitted energy at each position of the beam.

For STIM, the energy loss is converted to a thickness (areal density in $\mathrm{g} . \mathrm{cm}^{-2}$ ) assuming that the object has a global uniform composition (considering the main chemical elements) and that only its density varies. This assumption appears to be suited for a wide variety of 
microscopic dehydrated biological samples, which are mainly composed of organic matter, that's to say carbon, oxygen, nitrogen and hydrogen [25, 32]. For samples inhomogeneous in composition, this assumption would remain suitable as far as the variations of stopping power of the incoming beam remain negligible, which is usually the case for light element compounds [33]. For PIXE, the projection sinogram is obtained by sorting the X-rays according to their energy, which is specific to the emitting element. The number of X-rays at each position of the beam gives access to the element content, knowing the X-ray production cross section [27]. In this preliminary study, X-ray signal attenuation was not taken into account in the reconstruction process, so it is expected to give accurate results (compared to the numerical phantom) for thin samples only.

The final step of data reduction [27] produces the reconstructed images, which consists in a quantitative distribution of the mass density (in $\mathrm{g} . \mathrm{cm}^{-3}$ ) for STIM and of the element content (in $\mathrm{g} . \mathrm{cm}^{-3}$ ) for PIXE (one image per element). The images are recorded as binary raw data files, containing the density values in the pixels - or voxels - which can be read by most image processing software packages.

\subsection{Tomographic reconstruction}

The reconstruction was performed using the TomoRebuild software package, developed for STIM and PIXE tomography [27]. Two algorithms were implemented:

- Filtered Back Projection (FBP), based on the exact analytical solution of the continuous description of the tomography problem, often preferred as a simple and fast method suited for not too noisy data, especially transmission tomography data. A basic ramp filter was used.

- Maximum Likelihood Expectation Maximization (MLEM), an iterative method based on a discrete formulation of the tomography problem, often used for PIXE tomography as a robust algorithm even with noisy and/or incomplete data [5, 6, 34]. The images presented here were obtained from 32 iterations, which was the optimal number determined from NAAD and NRSMD calculations (see section 2.8).

\subsection{Image analysis}

We used the open-source ImageJ (https://imagej.nih.gov/ij) [35] image processing tool, developed by the National Institutes of Health, for the quantitative analysis of the images: shape and size of the different regions of the phantom, average density calculation and profiles. The 3D images were displayed using the Amira ${ }^{\circledR}$ software, from Thermo Fischer Scientific (https://www.fei.com/software/amira-for-life-sciences). The reconstructed images were compared to the original phantom, taken as a reference, at two levels: global and local.

The global discrepancy was evaluated using two different parameters: the Normalized Average Absolute Deviation (NAAD) and the Normalized Root Mean Squared Deviation (NRMSD), defined as follows:

$$
N A A D=\frac{\sum_{i=0}^{N-1}\left|I_{\text {ref }}(i)-I(i)\right|}{\sum_{i=0}^{N-1}\left|I_{\text {ref }}(i)\right|}
$$




$$
N R M S D=\sqrt{\frac{\sum_{i=0}^{N-1}\left(I_{\text {ref }}(i)-I(i)\right)^{2}}{\sum_{i=0}^{N-1}\left(I_{\text {ref }}(i)\right)^{2}}}
$$

In these expressions, $I$ stands for the reconstructed image, $I_{r e f}$ for the reference image and $i$ is the pixel index, in our case going from 0 to $N-1$, with $N=128 \times 128$. The C. elegans original phantom defined in Geant 4 was converted into a 3D voxelized phantom in order to make this comparison (the slice of interest was extracted from the voxelized volume) using a Python program. The problem of partial volume effects was taken into account at the boundary of the ellipsoids, so that the density values in these regions are intermediate between the inner part of the ellipsoid and the outer part. For this, the density values of the final $128 \times 128 \times 128$ voxelized phantom were defined from a temporary super-resolved image, composed of $256 \times 256 \times 256$ voxels. In the final image, we assign to the density value of each voxel the average of the content of the 8 voxels taken from the super-resolved image.

Image analysis at the local level was performed to evaluate the accuracy of the reconstructed density compared to the phantom, in different ROI (defined in the following figures). A profile through the cubic phantom was also studied to compare the edge sharpness between the two reconstruction methods.

\section{Results}

\subsection{STIM tomography on the cubic phantom}

Simulated data from a $5 \mu \mathrm{m}$ cubic phantom composed of phosphorus of homogenous density $\left(440 \mathrm{mg} . \mathrm{cm}^{-3}\right)$ were reconstructed, using different number of protons. Image analysis was performed on the central slice of the cube (Fig. 3a). The FBP reconstruction showed nonphysical negative density values outside the cube (Fig. $3 b$ ), which are inherent to this reconstruction method. A low threshold may be used to remove this background (Fig. $3 c$ ). The resulting image is then very similar to the MLEM reconstruction result (Fig. $3 d$ ), for which density values are intrinsically constrained to be positive. The average density (Table 3) was calculated from a ROI avoiding the sides of the cube (Fig. 3b), in order to discard partial volume effects that could alter the calculation. Very similar results were obtained for 100 and 1000 protons per shot, and are in agreement with the reference value, the relative error being below $2 \%$, for both reconstruction methods. On the profiles made across the cube (Fig. 4), the negative values outside the object are conspicuous for the FBP method, whereas for MLEM these voxels are exactly at zero. However, FBP shows a better definition of the edges (arrows in graph Fig. 4). The partial volume effects affect only one pixel at the border of the cube for FBP, whereas this transition region extends on \pm 2 pixels for MLEM.

\subsection{STIM tomography of the C. elegans phantom}

The 3D distribution of mass density was reconstructed (Fig. $5 a$ ) as well as the slice of interest (defined in Fig. 1), obtained from 5, 20, 100 and 1000 protons per shot (Fig. $5 b$ to $e$ ). The corresponding slice extracted from the voxelized phantom is also displayed for comparison (Fig. 5f). Similar results were found using the FBP and MLEM methods. The main differences compared to the phantom appear at the edges of the different regions, due to partial volume effects - and resulting high frequency effects for FBP (Fig. S3 in Supplementary material). As we have already seen for the cubic phantom, MLEM offers the advantages of discarding negative values and giving a background exactly at zero outside the 
object. However, it appears more sensitive to density variations at the edges (Fig. S4 in Supplementary material).

A question that we wanted to answer by designing these phantoms was to know whether the tomographic reconstruction process was able to give an accurate density determination in the different regions explored, when compared to the numerical phantom. The average density was calculated in 6 different ROI and compared, for different number of protons, to the reference density of the original Geant4 phantom (Fig. 6). The reconstructed density is in agreement with the reference value whatever the ROI or the chosen number of protons, including for ROI 1 (vacuum outside the object). The statistical fluctuations of density decrease when the number of protons increases (also visible directly in the images Fig. 5).

It is important, in order to minimize the duration of the simulations (and also of the real experiments), to determine what would be the minimal number of protons that should be used. For this, we plotted the NAAD and NRMSD evaluation of the global relative error as a function of the number of protons per shot (Fig. 7). For the two parameters, the relative error between the reconstructed and the reference phantom images decreases when the number of protons increases. However, the improvement is lesser for high numbers of protons: for instance, it is conspicuous when going from 5 to 20 protons per shot, corresponding to a $46 \%$ decrease for NAAD, whereas there is very little improvement when going from 100 to 1000 protons (with only $5 \%$ decrease). The NAAD parameter was preferred for this quantification because it varies more quickly according to the number of protons.

\subsection{PIXE tomography}

Keeping in mind that, for this study, X-ray signal attenuation was not taken into account in the reconstruction process, we first analyzed a thin sample where X-ray attenuation is negligible. The average density was calculated in the ROI defined in Fig. 3 for the $5 \mu \mathrm{m}$ cubic phantom (Table 4). As expected, the results are in agreement with the reference value for both reconstruction methods. To test the effect of X-ray signal attenuation, the calculation was repeated for a $40 \mu \mathrm{m}$ cubic phantom having the same density and composition as the "body" part of the $C$. elegans phantom. The decrease of ionization cross section, resulting in a decrease in X-ray production, and the X-ray absorption within the sample have been already estimated for this type of sample [27]. As expected, since the reconstruction algorithm does not take into account these attenuation effects, the calculated density was underestimated. A similar behavior was observed for the $C$. elegans phantom for all reconstructed elements. Only the results for phosphorus were reported in Table 4: this element was chosen because it is the most attenuated. FBP and MLEM results were found very similar (also from a quantitative point of view) for all elements. The MLEM density distribution maps are displayed for all the reconstructed elements (Fig. 8). Statistical fluctuations are lesser for MLEM (as shown in Table 4). Note that the ramp filter is still used here for FBP, for comparison purposes, whereas it is the most penalizing considering high frequency statistical fluctuations in the image. This effect could have been reduced by using a smoothing filter instead.

\section{Discussion}

One of the questions we wanted to answer in this study was to determine the optimal number of protons per shot that is required to obtain an accurate determination of density for STIM tomography. We consider here only the effect of reconstruction process, taking the numerical 
Geant 4 phantom as a reference. The results presented in section 3.2 and especially Fig. 7 lead us to choose an optimal number of incident protons of 20 for 3D imaging (we could go up to 100 for individual slices). This represents a good compromise to minimize the duration of simulations whilst maintaining a good quality image, from a visual point of view as well as for quantitative determination. This is in agreement with the results obtained for classical 2D STIM for microscopic samples [31]. Using 20 protons per shot, the global error on the whole image (NAAD, Fig. 7) is lower than $10 \%$ for the STIM tomography of the C. elegans phantom. Moreover, the accuracy in determining the average density in the different ROI ranges from 0.7 to $2 \%$ according to the ROI (Fig. 6).

To make a comparison with macroscopic pCT, Sadrozinski et al. [36] concluded that stable solutions could be reached from 25 protons in the final considered events. This corresponds to about 100 protons per shot, taking into account that about $75 \%$ of the incident protons are discarded during data processing: a first loss is due to inelastic interactions in the phantom (about 50\%); an additional loss of 50\% results from the fact that only the leading edge of the residual energy distribution is fitted using a Gaussian function, in order to get the most probable value. None of these phenomena occur for STIM tomography, as multiple scattering is negligible and no Gaussian fit is made.

\section{Conclusion}

We designed a Geant4 simulation for 3D proton imaging at microscopic level using STIM and PIXE tomography. The code (including the Python program converting the $C$. elegans geometry into a voxelized phantom) is intended to be open-source, and can be included in Geant 4 for diffusion to its users' community. A detailed user guide in English is available to describe all the steps of the simulation, from the phantom design to the construction of the macros and Python scripts. The duration of the simulation makes parallel computing unavoidable, at least for PIXE tomography, considering the high number of incident protons required at each position of the beam. Further studies could improve the simulation duration for PIXE tomography by porting our Geant 4 simulation to GPU architecture.

The reconstruction algorithms applied on simulated data lead to distributions of mass density, either global (STIM) or for each analyzed element (PIXE). For STIM, the reconstructed density values are in good agreement with the expected reference values from the numerical phantom. An optimal number of 20 protons per shot was obtained to produce good quality 3D STIM images (from visual and also quantitative points of view).

For PIXE tomography, a good agreement was found for the thin sample only (the $5 \mu \mathrm{m}$ cubic phantom), whereas, as expected, the element density was underestimated for thick samples (the $40 \mu \mathrm{m}$ cube and the modified $C$. elegans phantoms). These preliminary results on thick samples confirm the need to correct for X-ray signal attenuation during the reconstruction process. For future studies, we would like to use iterative reconstruction methods, which enable including correction models for X-ray signal attenuation, in order to obtain a more accurate reconstruction of PIXE data. We would like to emphasize that he Geant 4 simulation will constitute a reliable reference to check for the accuracy of such correction, for two main reasons. First, the Geant 4 simulation provides all the required information (energy, direction of the emitted X-rays) that will enable such checking. A second important fact is that the Geant 4 simulation is obviously totally independent of the reconstruction algorithm. That brings an interesting advantage compared to previous PIXE tomography studies, in which the accuracy of the results was checked either by cross-comparison of different reconstruction 
algorithms or by projecting simulated data using the same (reversed) algorithm as the one used for reconstruction.

\section{Acknowledgements}

The authors are grateful to Laurent Daudin, Stéphanie Sorieul, Philippe Alfaurt, Franck Delalée and Laurent Sérani, Centre d'Etudes Nucléaires de Bordeaux Gradignan, France, for their support in the technical developments on the tomography beamline. The authors wish to express their thanks to the Caenorhabditis Genetics Center (CGC), University of Minnesota, for providing C. elegans (N2 Bristol) and E. coli OP50 strains. CGC is funded by NIH Office of Research Infrastructure Programs (P40 OD010440). This work has been financially supported by the CNRS "Mission pour les Initiatives Transverses et Interdisciplinaires" (MITI) in the frame of the "Appel à Projets Interne Inter-Institut 2018", "Simulation Numérique pour le Traitement des Images de tomographie par émission X" - NuméTriX project (CENBG, IMS, LaBRI). Tomography experiments on $C$. elegans have been carried out in the frame of the ANR Program: "Contaminants, Ecosystèmes, Santé 2010" TITANIUMS project. The Région Nouvelle Aquitaine has supported financially the AIFIRA facility and the technical development of the microbeam line, as also the development of biological protocols through the TOX-NANO program (contract $\mathrm{n}^{\circ}$ 20111201003). Tomography experiments were partly supported by the European Community as an Integrating Activity "Support of Public and Industrial Research Using Ion Beam Technology (SPIRIT)" under EC contract $\mathrm{n}^{\circ} 227012$ and as an "Integrating Activity Supporting Postgraduate Research with Internships in Industry and Training Excellence" (SPRITE) under EC contract $\mathrm{n}^{\circ} 317169$.

\section{References}

[1] Cormack M, Koehler AM. Quantitative proton tomography: preliminary experiments. Phys. Med. Biol. 1976;21(4):560-569. https://doi.org/10.1088/0031-9155/21/4/007

[2] Michelet C, Barberet P, Moretto P, Seznec H. Development and applications of STIMand PIXE-tomography: a review. Nucl. Instr. Meth. B 2015;363:55-60. https://doi.org/10.1016/j.nimb.2015.08.070

[3] Michelet C, Barberet P, Desbarats P, Giovannelli J-F, Schou C, Chebil I et al. An implementation of the NiftyRec medical imaging library for PIXE-tomography reconstruction. Nucl. Instr. Meth. B 2017;404:131-139. https://dx.doi.org/10.1016/j.nimb.2017.01.067

[4] Guo N, Lu H.Y, Wang Q, Meng J, Gao DZ, Zhang YJ et al. A dual-PIXE tomography setup for reconstruction of Germanium in ICF target. Nucl. Instr. Meth. B 2017;404:162-166. https://dx.doi.org/10.1016/j.nimb.2017.04.041

[5] Beasley DG, Alves LC, Barberet Ph, Bourret S., Devès G, Gordillo N. et al. A comparison of quantitative reconstruction techniques for PIXE-tomography analysis applied to biological samples. Nucl. Instr. Meth. B 2014;331:248-252. https://dx.doi.org/10.1016/j.nimb.2015.08.070

[6] Satoh T. Yokoyama A, Kitamura A, Ohkubo T, Ishii Y, Takahatake Y et al. Particle induced X-ray emission-computed tomography analysis of an adsorbent for extraction chromatography. Nucl. Instr. Meth. B 2016;371:419-423. https://dx.doi.org/10.1016/j.nimb.2015.09.076 
[7] Lu HY, He XD, Meng J, Guo N, Rong CC, Zhang W et al. Reconstruction of Ge spatial distribution in ICF target using PIXE-T. Fusion Engineering and Design 2016;113:4350. https://dx.doi.org/10.1016/j.fusengdes.2016.10.006

[8] Agostinelli S et al., by the Geant4 Collaboration. Geant4 - a simulation toolkit. Nucl. Instr. Meth. A 2003;506:250-303. https://dx.doi.org/10.1016/S0168-9002(03)01368-8

[9] Allison J et al., by the Geant4 Collaboration. Geant4 developments and applications. IEEE Trans. Nucl. Sci. 2006;53(1):270-278. https://dx.doi.org/10.1109/TNS.2006.869826

[10] Allison $\mathbf{J}$ et al., by the Geant4 Collaboration. Recent developments in Geant4. Nucl. Instr. Meth. A 2016;835:186-225. https://doi.org/10.1016/j.nima.2016.06.125

[11] Sechopoulos I, Rogers DWO, Bazalova-Carter M, Bolch WE, Heath EC, McNitt-Gray $\mathrm{MF}$ et al. RECORDS: improved Reporting of montE CarlO RaDiation transport Studies: Report of the AAPM Research Committee Task Group 268. Med. Phys. 2018;45 (1):e1-e5. https://dx.doi.org/10.1002/mp.12702

[12] Arbor N, Dauvergne D, Dedes G, Létang JM, Parodi K, Quiñones CT et al. Monte Carlo comparison of $\mathrm{x}$-ray and proton $\mathrm{CT}$ for range calculations of proton therapy beams. Phys. Med. Biol. 2015;60:7585-7599. https://dx.doi.org/10.1088/00319155/60/19/7585

[13] Ardenfors O, Henry T, Gudowska I, Poludniowski G, Dasu A. Organ doses from a proton gantry-mounted cone-beam computed tomography system characterized with MCNP6 and GATE. Phys. Med. 2018;53:56-61. https://doi.org/10.1016/j.ejmp.2018.08.011

[14] Giacometti V, Guatelli S, Bazalova-Carter M, Rosenfeld AB, Schulte RW. Development of a high resolution voxelised head phantom for medical physics applications. $\quad$ Phys. Med. 2017;33:182-188. https://dx.doi.org/10.1016/j.ejmp.2017.01.007

[15] Bashkirov VA, Johnson RP, Sadrozinski HF-W, Schulte RW. Development of proton computed tomography detectors for applications in hadrontherapy. Nucl. Instr. Meth. A 2016; 809:120-129. https://dx.doi.org/10.1016/j.nima.2015.07.066

[16] Pettersen HES, Alme J, Barnaföldi GG, Barthel R, van den Brink A, Chaar M et al. Design optimization of a pixel-based range telescope for proton computed tomography. Phys. Med. 2019;63:87-97. https://doi.org/10.1016/j.ejmp.2019.05.026

[17] Cirrone GAP, Bucciolini M, Bruzzi M, Candiano G, Civinini C, Cuttone G et al. Monte Carlo evaluation of the Filtered Back Projection method for image reconstruction in proton computed tomography. Nucl. Instr. Meth. A 2011; 658:78-83. https://dx.doi.org/10.1016/j.nima.2011.05.061

[18] Biegun AK, van Goethem MJ, van der Graaf ER, van Beuzekom M, Koffeman EN, Nakaji et al. The optimal balance between quality and efficiency in proton radiography imaging technique at various proton beam energies: A Monte Carlo study. Phys. Med. 2017;41:141-146. https://dx.doi.org/10.1016/j.ejmp.2017.08.006

[19] Incerti S, Barberet Ph, Devès G, Michelet C, Francis Z, Ivantchenko V et al. Comparison of experimental proton-induced fluorescence spectra for a selection of thin high-Z samples with Geant4 Monte Carlo simulations. Nucl. Instr. Meth. B 2015;358:210-222. https://doi.org/10.1016/j.nimb.2015.06.029

[20] Apostolakis J, Giani S, Maire M, Nieminen P, Pia MG, Urban L. Geant4 low energy electromagnetic models for electrons and photons. INFN/AE-99/18 September 1999;115. https://dx.doi.org/ 10.15161/oar.it/1448986129.49

[21] Bearden JA. X-Ray wavelengths. Rev. Mod. Phys. 1967;39:78-124. https://doi.org/10.1103/RevModPhys.39.78 
[22] Le Trequesser Q, Saez G, Devès G, Michelet C, Barberet P, Delville MH, Seznec H. In situ titanium dioxide nanoparticles quantitative microscopy in cells and in C. elegans using nuclear microprobe analysis. Nucl. Instr. Meth. B 2014;341(15):58-64. https://doi.org/10.1016/j.nimb.2014.06.031

[23] Le Trequesser Q, Devès G, Saez G, Daudin L, Barberet P, Michelet C, Delville MH, Seznec H. Single cell in situ detection and quantification of metal oxide nanoparticles using multimodal correlative microscopy. Anal Chem. 2014;86(15):7311-7319. https://doi.org/10.1021/ac501318c

[24] Muggiolu G, Simon M, Devès G, Barberet P, Michelet C, Delville MH, Seznec H. In Situ Detection and Single Cell Quantification of Metal Oxide Nanoparticles using Nuclear Microprobe Analysis. J Vis Exp. 2018;132:e55041. https://doi.org/10.3791/55041.

[25] Carmona A, Devès G, Ortega R. Quantitative micro-analysis of metal ions in subcellular compartments of cultured dopaminergic cells by combination of three ion beam techniques. Anal Bioanal Chem. 2008;390:1585-1594. https://doi.org/10.1007/s00216-008-1866-6

[26] Habchi C, Gordillo N, Bourret S, Barberet Ph, Jovet C, Moretto Ph et al. Beyond filtered backprojection: a reconstruction software package for ion beam microtomography data, Nucl. Instr. Meth. B 2013;295:42-49. https://doi.org/10.1016/j.nimb.2012.10.006

[27] Michelet C, Barberet Ph, Devès G, Bouguelmouna B, Bourret S, Delville M-H et al. Quantitative reconstruction of PIXE-tomography data for thin samples using GUPIX Xray emission yield. Nucl. Instr. Meth. B 2015;348:92-99. https://doi.org/10.1016/j.nimb.2014.11.035

[28] Devès G, Ortega R. Subcellular mass determination by ${ }^{4} \mathrm{He}^{+}$energy-loss microspectrometry. Anal Bioanal Chem. 2002;374:390-394. https://doi.org/10.1007/s00216002-1511-8

[29] Devès G, Isaure MP, Le Lay P, Bourguignon J, Ortega R. Fully quantitative imaging of chemical elements in Arabidopsis thaliana tissues using STIM, PIXE and RBS. Nucl. Instr. Meth. B 2005;231:117-122. https://doi.org/ 10.1016/j.nimb.2005.01.044

[30] Okada S, Murakami K, Amako K, Sasaki T, Incerti S, Karamitros M et al. GPU Acceleration of Monte Carlo Simulation at the Cellular and DNA Levels. Smart Innovation, Systems and Technologies 2016;45:323-332. https://doi.org/10.1007/978-3319-23024-5_29

[31] Overley JC, Schofield RMS, MacDonald JD, Lefevre JH. Energy-loss image formation in scanning transmission ion microscopy. Nucl. Instr. Meth. B 1988;30:337-341. https://doi.org/10.1016/0168-583X(88)90022-5

[32] Snyder WS, Cook MJ, Nasset ES, Karhausen LR, Parry Howells GP, Tipton IH. Report of the task group on reference man. ICRP Publication 23, 1975, Pergamon Press, Oxford. http://www.icrp.org/publication.asp?id=ICRP\%20Publication\%2023

[33] Lefevre HW, Schofield RMS. Scanning transmission ion microscopy as it complements particle induced X-ray emission microanalysis. Scanning Microscopy 1987;1:879-889. PMID:3116664

[34] Ng YK, Orlic I, Liew SC, Loh KK, Tang SM, Osipowicz T et al. A PIXE microtomography experiment using MLEM algorithm. Nucl. Instr. Meth. B 1997;130(14):109-112. https://doi.org/10.1016/S0168-583X(97)00303-0 
[35] Rueden C T, Schindelin J, Hiner M C et al. ImageJ2: ImageJ for the next generation of scientific image data. BMC Bioinformatics 2017; 18:529. https://doi.org/doi:10.1186/s12859-017-1934-Z

[36] Sadrozinski HF-W, Johnson RP, Macafee S, Plumb A, Steinberg D, Zatserklyaniy A et al. Development of a head scanner for proton CT. Nucl. Instr. Meth. A 2013;699:205210. https://dx.doi.org/10.1016/j.nima.2012.04.029

\section{Tables}

\section{Table 1}

Geometrical parameters of the six ellipsoids used to model the shape of the C. elegans phantom. The size and position of the ellipsoids were derived from STIM tomography experimental data obtained at CENBG [26, 27].

\section{Table 2}

Content of the six ellipsoids of the $C$. elegans phantom. The ellipsoids have a uniform mass density and composition (here expressed in percentage by mass, as defined in the Geant 4 material composition). The values were derived from experimental STIM (mass density) and PIXE (element content) tomography data obtained at CENBG [26, 27].

\section{Table 3}

Average density obtained from the reconstruction of simulated data of the cubic phantom, from the ROI defined in Fig. $3 b$, using 100 or 1000 protons per shot. The given uncertainty corresponds to the standard deviation in the ROI.

\section{Table 4}

Average density of phosphorus, obtained from the reconstruction of PIXE tomography simulated data of the cube and the $C$. elegans phantoms, without taking into account X-ray signal attenuation in the reconstruction process. The given uncertainty corresponds to the standard deviation in the ROI. For $C$. elegans, the ROI considered here are not those defined in Fig. 6 but correspond to the segmented regions of the phantom (in order to increase statistics and also to get a value for the cuticle).

\section{Figure captions}

Fig. 1. Schematic view of the C. elegans phantom, showing the six ellipsoids used to model the different parts of the worm (left and bottom right). The ROI at the beginning of intestine (upper right) and the specific slice going through the cell nuclei and the Ti rich region are displayed using Geant4 visualization.

Fig. 2. Geometry of the scan: the position of the source follows a 2D grid (represented on the left). The particular example represented here would result in 5 horizontal tomographic slices of $4 \times 4$ pixels each.

Fig. 3. Reconstruction of the STIM simulated data for the cubic phantom using 1000 protons per shot. The 3D image (a) was displayed using an isosurface value close to zero, corresponding to the boundary between the outer surface of the cube and surrounding vacuum. The central slice is displayed, as obtained by FBP without $(b)$ and with $(c)$ low 
density threshold, and by MLEM $(d)$. For the slices, a linear grey scale was used to code mass density values.

Fig. 4. Profile through the cubic phantom (upper left arrow), comparing the reconstructed images (FBP, MLEM) to the original Geant4 phantom (Reference). The calculated mass density is displayed according to the position along the 128 pixels of the profile (from -4.5 to $+4.5 \mu \mathrm{m}$ ). In this case, the reference density values are constant, at $440 \mathrm{mg} . \mathrm{cm}^{-3}$ within the cube and 0 outside.

Fig. 5. FBP reconstruction of the STIM tomography simulated data of the C. elegans phantom. The 3D distribution $(a)$ is displayed using a color and transparency scale going from transparent dark red (lowest density) to opaque yellow (highest density). The slices obtained from 5, 20, 100 and 1000 protons per shot $(b-e)$ are displayed with no additional filter (except the ramp filter used for FBP) nor threshold, so that they can be directly compared to the voxelized version of the original phantom $(f)$.

Fig. 6. Average mass density obtained from FBP reconstruction in 6 regions of interest of the C. elegans phantom from 5, 20, 100 and 1000 protons per shot, compared to the original Geant 4 phantom (reference). The error bars correspond to the standard deviation. The same ROI were applied for all images (as indicated upper left). The ROI correspond to the vacuum outside the object (ROI 1 - the mass density on the vertical axis is also in $\mathrm{mg} . \mathrm{cm}^{-3}$ ), the body of the worm (ROI 2), the first and second cell nuclei (ROI 3 and 4), the intestine (ROI 5), the Ti rich region inside the intestine (ROI 6).

Fig. 7. NRSMD and NAAD evaluation of the relative error between the reconstructed FBP image (ramp filter) and the voxelized version of the $C$. elegans phantom, taken as a reference. The horizontal scale representing the number of protons was interrupted between 100 and 1000 for clarity, as there is very little variation between these two points.

Fig. 8. MLEM reconstruction of the PIXE tomography simulated data for the C. elegans phantom, for each considered chemical element: $\mathrm{P}, \mathrm{S}, \mathrm{Cl}, \mathrm{K}, \mathrm{Ca}$, Ti. The mass density calculated for each element is coded on a linear grey scale going from the lowest value (zero, in black) to the highest, as indicated.

\section{Appendix A. Supplementary data}

Supplementary Fig. S1. Example of transmitted energy spectrum, corresponding to a 1.5 $\mathrm{MeV}$ incident proton beam going through the middle of the slice of the C. elegans phantom defined in Fig. 1. The spectrum obtained from 1000 incident protons is displayed, with or without the $\pm 7^{\circ}$ angular limitation on the direction of the transmitted protons. The two spectra are almost identical; their difference is displayed below for clarity.

Supplementary Fig. S2. X-ray energy spectrum obtained from the $C$. elegans phantom in a $4 \pi$ solid angle, for $10^{6}$ incident protons per shot.

Supplementary Fig. S3. Difference images (Reference phantom - Reconstructed image) obtained from FBP and MLEM reconstruction of simulated data for 20 and 1000 protons per shot. The same linear grey scale was used for all images for comparison purpose, going from the lowest difference of density $\left(-0.29 \mathrm{~g} . \mathrm{cm}^{-3}\right.$, in black) to the highest $\left(+0.44 \mathrm{~g} . \mathrm{cm}^{-3}\right.$, in white $)$. The difference values were within the intervals: [-0.29; 0.38] for FBP 20 protons, [-0.28; 
0.35] for FBP 1000 protons, [-0.27; 0.44] for MLEM 20 protons and [-0.28; 0.44] for MLEM 1000 protons.

Supplementary Fig. S4. Example of profiles of the difference images (Reference phantom Reconstructed image) obtained from FBP and MLEM reconstruction of simulated data for 1000 protons per shot. The line selected for this profile is represented upper left. The discrepancy between the density values relative to the reference phantom is slightly higher at the edges for MLEM than for FBP. 
Table 1

\begin{tabular}{|l|c|l|l|l|l|l}
\hline Region & $\begin{array}{l}\text { Ellipsoid } \\
\text { number }\end{array}$ & $\begin{array}{l}\text { Semi-axis } \\
\text { in } x \\
\text { direction } \\
(\mu \mathrm{m})\end{array}$ & $\begin{array}{l}\text { Semi-axis } \\
\text { in } y \\
\text { direction } \\
(\mu \mathrm{m})\end{array}$ & $\begin{array}{l}\text { Semi-axis } \\
\text { in z } \\
\text { direction } \\
(\mu \mathrm{m})\end{array}$ & $\begin{array}{l}\text { Position of the } \\
\text { center }(\mu \mathrm{m})\end{array}$ & $\begin{array}{l}\text { Rotation } \\
\text { around } z \\
\text { axis } \\
\text { (degree) }\end{array}$ \\
\hline Cuticle & $\mathbf{1}$ & 20.6 & 21.4 & 188 & $(0 ; 0 ; 0)$ & 0 \\
\hline Body & $\mathbf{2}$ & 18.6 & 19.0 & 187 & $(-0.39 ; 0 ; 0)$ & 0 \\
\hline Nucleus 1 & $\mathbf{3}$ & 1.95 & 3.23 & 4.32 & $(1.97 ;-7.09 ; 18.07)$ & 0 \\
\hline Nucleus 2 & $\mathbf{4}$ & 2.08 & 2.46 & 4.32 & $(8.27 ;-3.15 ; 18.07)$ & 0 \\
\hline Intestine & $\mathbf{5}$ & 3.67 & 16.5 & 28.7 & $(1.25 ; 0.61 ; 0)$ & -59 \\
\hline $\begin{array}{l}\text { Ti rich } \\
\text { region }\end{array}$ & $\mathbf{6}$ & 1.62 & 1.95 & 1.62 & $(6.25 ; 3.61 ; 18.07)$ & 0 \\
\hline
\end{tabular}


Table 2

\begin{tabular}{|c|c|c|c|c|c|c|c|c|}
\hline Region & $\mathbf{P}$ & $\mathbf{S}$ & CI & K & $\mathrm{Ca}$ & $\mathrm{Ti}$ & $\mathrm{C}_{10} \mathrm{H}_{17} \mathrm{O}_{3} \mathrm{~N}_{2}$ & $\begin{array}{c}\text { Total mass } \\
\text { density } \\
\left(\mathrm{mg} \cdot \mathrm{cm}^{-3}\right)\end{array}$ \\
\hline Cuticle & $0.47 \%$ & $0.21 \%$ & $0.05 \%$ & $0.48 \%$ & & & $98.8 \%$ & 497 \\
\hline Body & $0.72 \%$ & & & & & & $99.3 \%$ & 408 \\
\hline Nucleus 1 & $0.57 \%$ & & & & $1.5 \%$ & & $97.9 \%$ & 663 \\
\hline Nucleus 2 & $0.61 \%$ & & & & $1.4 \%$ & & $97.9 \%$ & 603 \\
\hline Intestine & & $0.12 \%$ & $0.02 \%$ & $0.20 \%$ & & & $99.7 \%$ & 541 \\
\hline $\begin{array}{l}\text { Ti rich } \\
\text { region }\end{array}$ & & $0.10 \%$ & $0.02 \%$ & $0.18 \%$ & & $0.05 \%$ & $99.6 \%$ & 751 \\
\hline
\end{tabular}


Table 3

\begin{tabular}{|c|c|c|c|c|c|}
\hline \multirow[b]{2}{*}{$\begin{array}{l}\text { Reconstruction } \\
\text { method }\end{array}$} & \multicolumn{2}{|c|}{100 protons } & \multicolumn{2}{|c|}{1000 protons } & \multirow[b]{2}{*}{$\begin{array}{l}\text { Reference } \\
\left(\mathbf{m g} \cdot \mathrm{cm}^{-3}\right)\end{array}$} \\
\hline & $\begin{array}{l}\text { Average } \\
\text { density } \\
\left(\mathrm{mg}^{\left.-\mathrm{cm}^{-3}\right)}\right.\end{array}$ & $\begin{array}{l}\text { Relative } \\
\text { error }\end{array}$ & $\begin{array}{l}\text { Average } \\
\text { density } \\
\left(\mathrm{mg}^{\left.-\mathrm{cm}^{-3}\right)}\right.\end{array}$ & $\begin{array}{l}\text { Relative } \\
\text { error }\end{array}$ & \\
\hline FBP & $435 \pm 42$ & $1.1 \%$ & $435 \pm 24$ & $1.1 \%$ & \multirow{2}{*}{440} \\
\hline MLEM & $438 \pm 21$ & $0.37 \%$ & $438 \pm 15$ & $0.41 \%$ & \\
\hline
\end{tabular}


Table 4

\begin{tabular}{|c|c|c|c|c|c|c|}
\hline & & \multicolumn{2}{|c|}{ FBP } & \multicolumn{2}{|c|}{ MLEM } & \multirow[b]{2}{*}{$\begin{array}{l}\text { Reference } \\
\text { density P } \\
\left(\mathrm{mg} \cdot \mathrm{cm}^{-3}\right)\end{array}$} \\
\hline & & $\begin{array}{l}\text { Average } \\
\text { density P } \\
\left(\mathrm{mg} \cdot \mathrm{cm}^{-3}\right)\end{array}$ & $\begin{array}{c}\text { Relative } \\
\text { error }\end{array}$ & $\begin{array}{l}\text { Average } \\
\text { density P } \\
\left(\mathrm{mg} \cdot \mathrm{cm}^{-3}\right)\end{array}$ & $\begin{array}{c}\text { Relative } \\
\text { error }\end{array}$ & \\
\hline \multirow{2}{*}{\multicolumn{2}{|c|}{$\begin{array}{l}\text { Cube } 5 \mu \mathrm{m} \\
\text { Cube } 40 \mu \mathrm{m}\end{array}$}} & $430 \pm 76$ & $-2.4 \%$ & $434 \pm 34$ & $-1.5 \%$ & 440 \\
\hline & & $19.0 \pm 6.1$ & $-36 \%$ & $19.5 \pm 3.3$ & $-35 \%$ & 29.5 \\
\hline \multirow{4}{*}{$\begin{array}{c}\text { C. } \\
\text { elegans }\end{array}$} & Cuticle & $18.4 \pm 4.7$ & $-22 \%$ & $19.5 \pm 3.6$ & $-17 \%$ & 23.4 \\
\hline & Body & $18.3 \pm 4.8$ & $-39 \%$ & $18.3 \pm 2.6$ & $-39 \%$ & 29.5 \\
\hline & Nucleus 1 & $18.4 \pm 4.8$ & $-51 \%$ & $17.8 \pm 2.1$ & $-53 \%$ & 37.5 \\
\hline & Nucleus 2 & $17.9 \pm 3.6$ & $-54 \%$ & $17.9 \pm 1.6$ & $-51 \%$ & 36.7 \\
\hline
\end{tabular}


Fig. 1

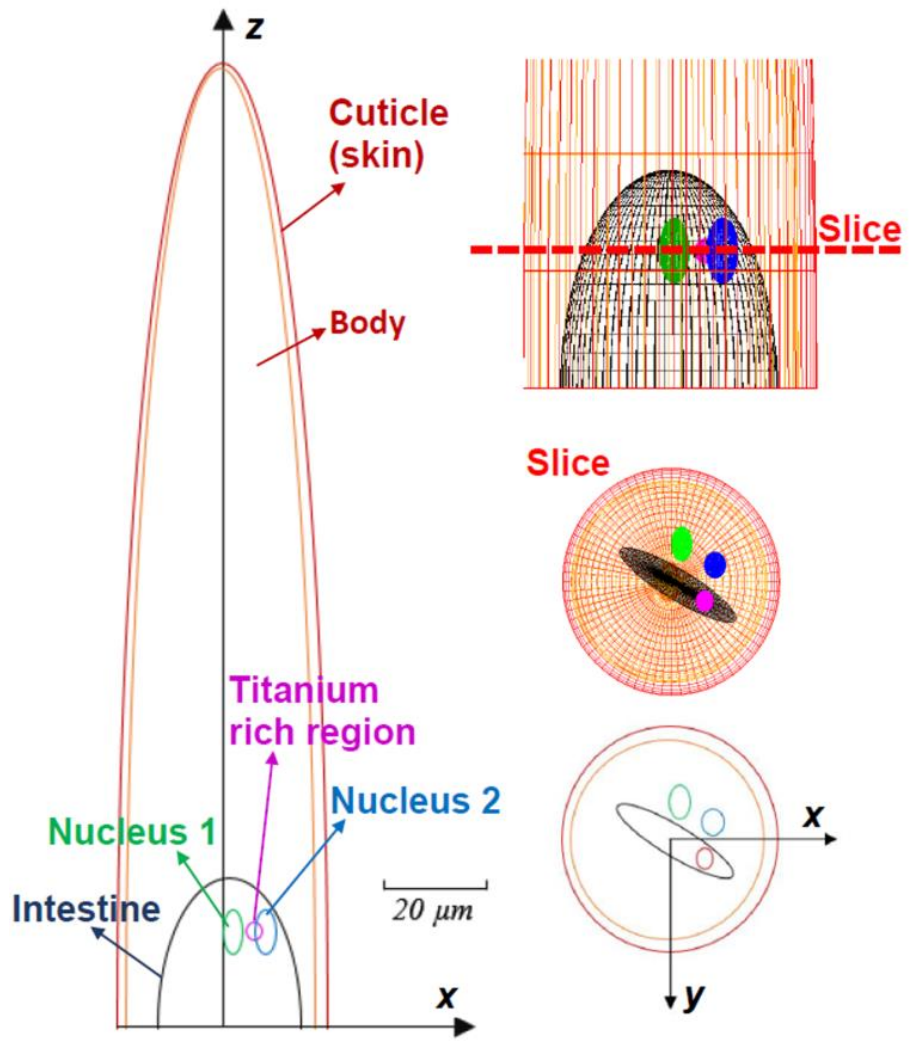


Fig. 2

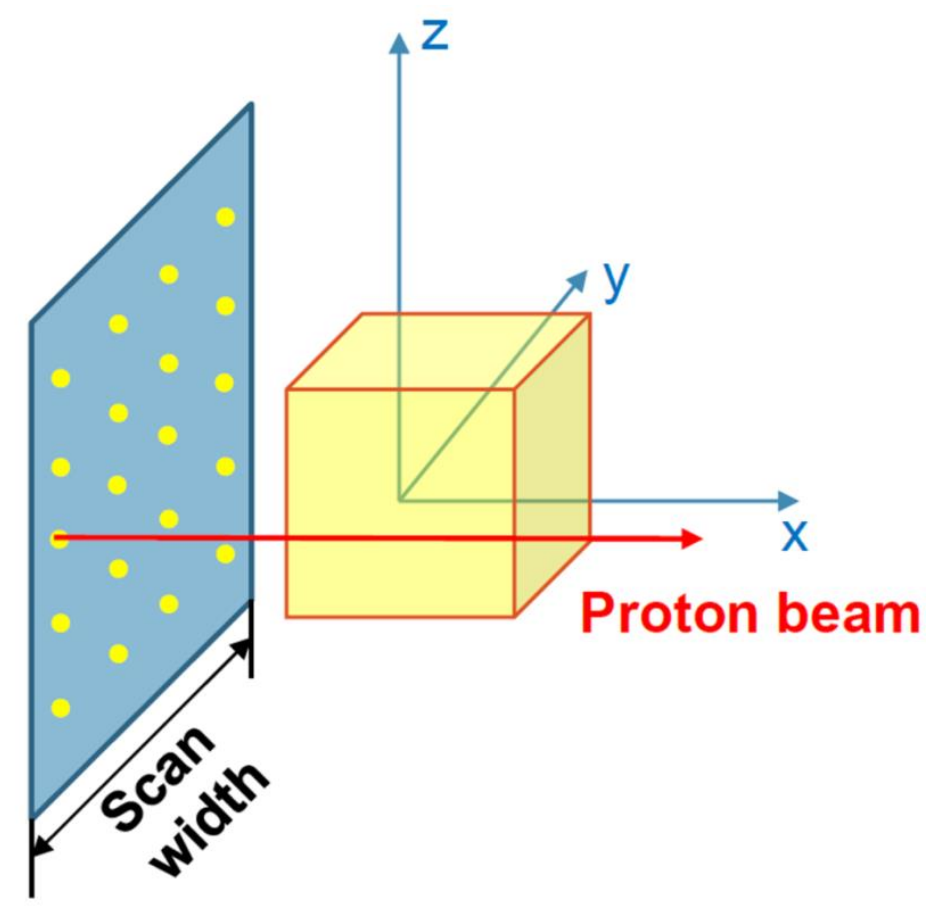


Fig. 3
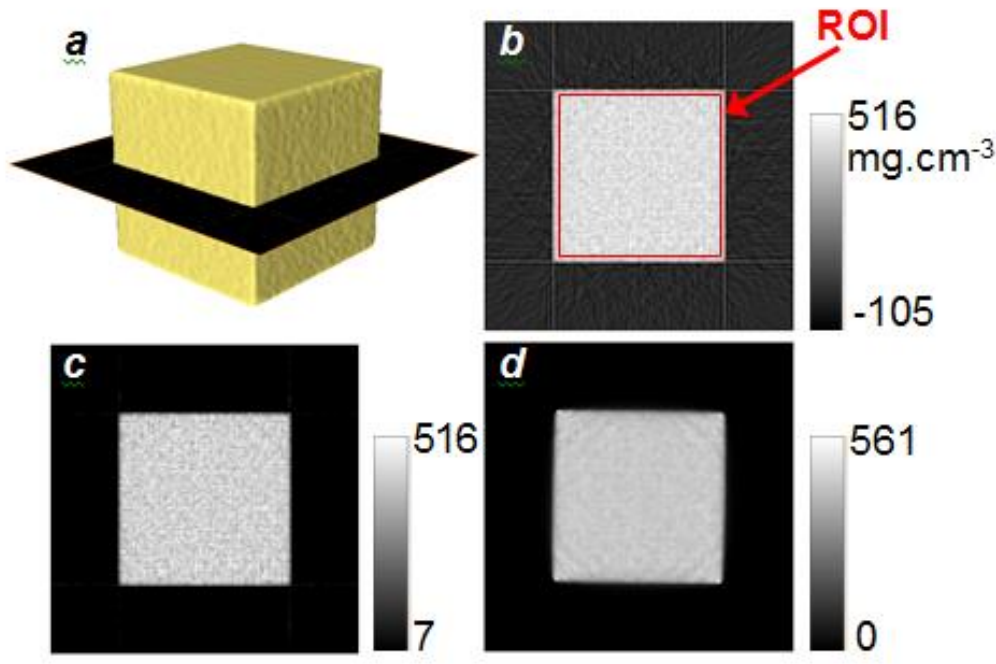
Fig.4

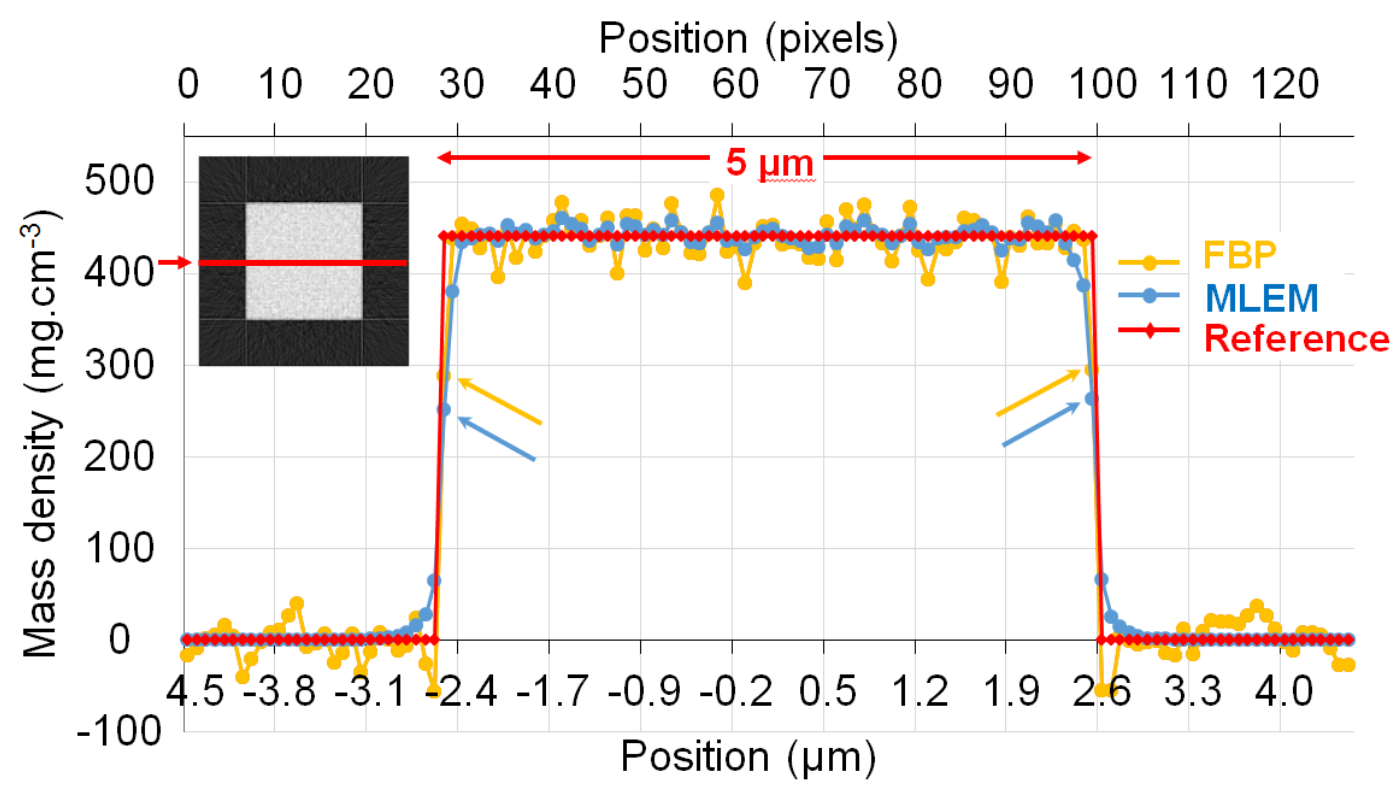


Fig. 5

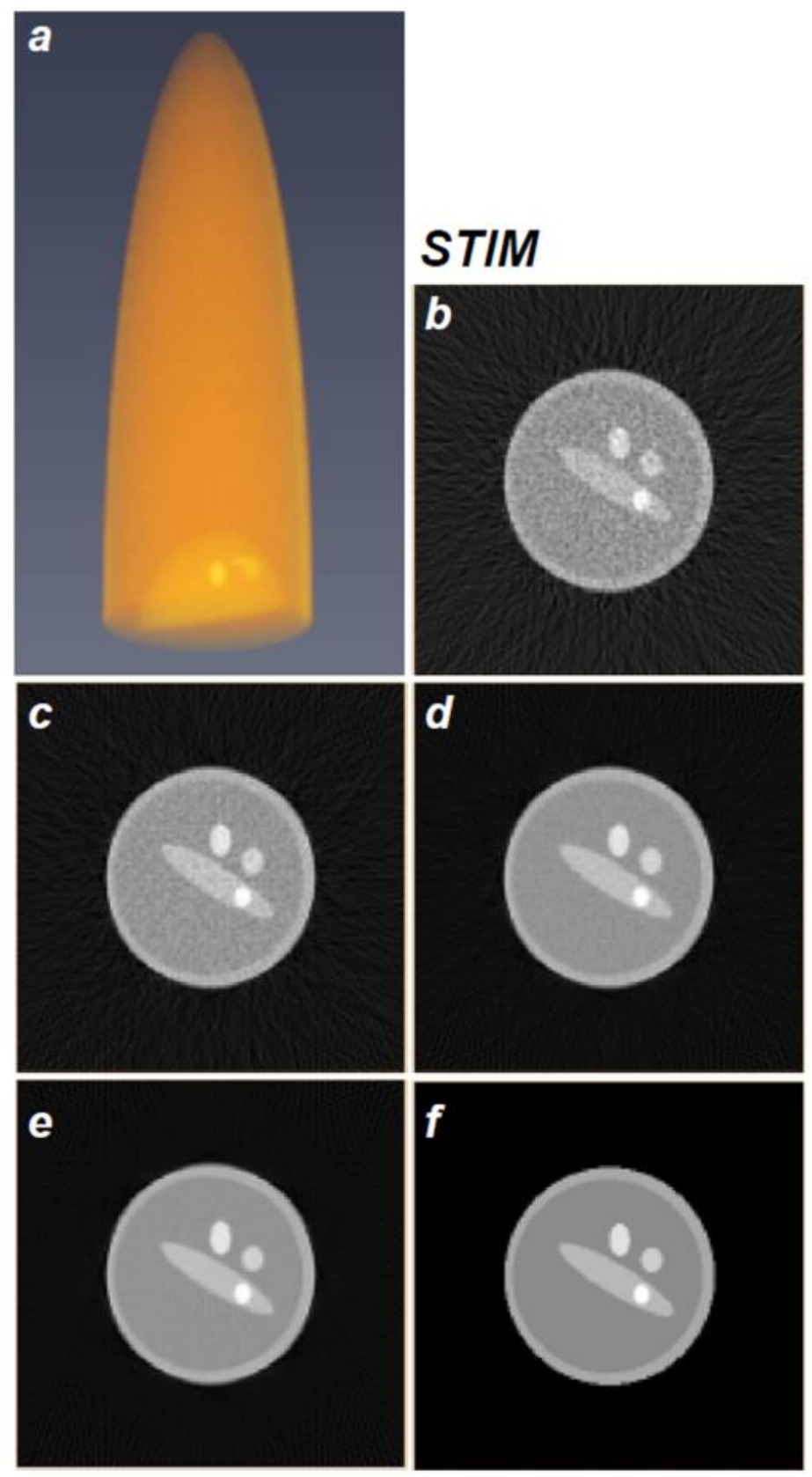


Fig. 6

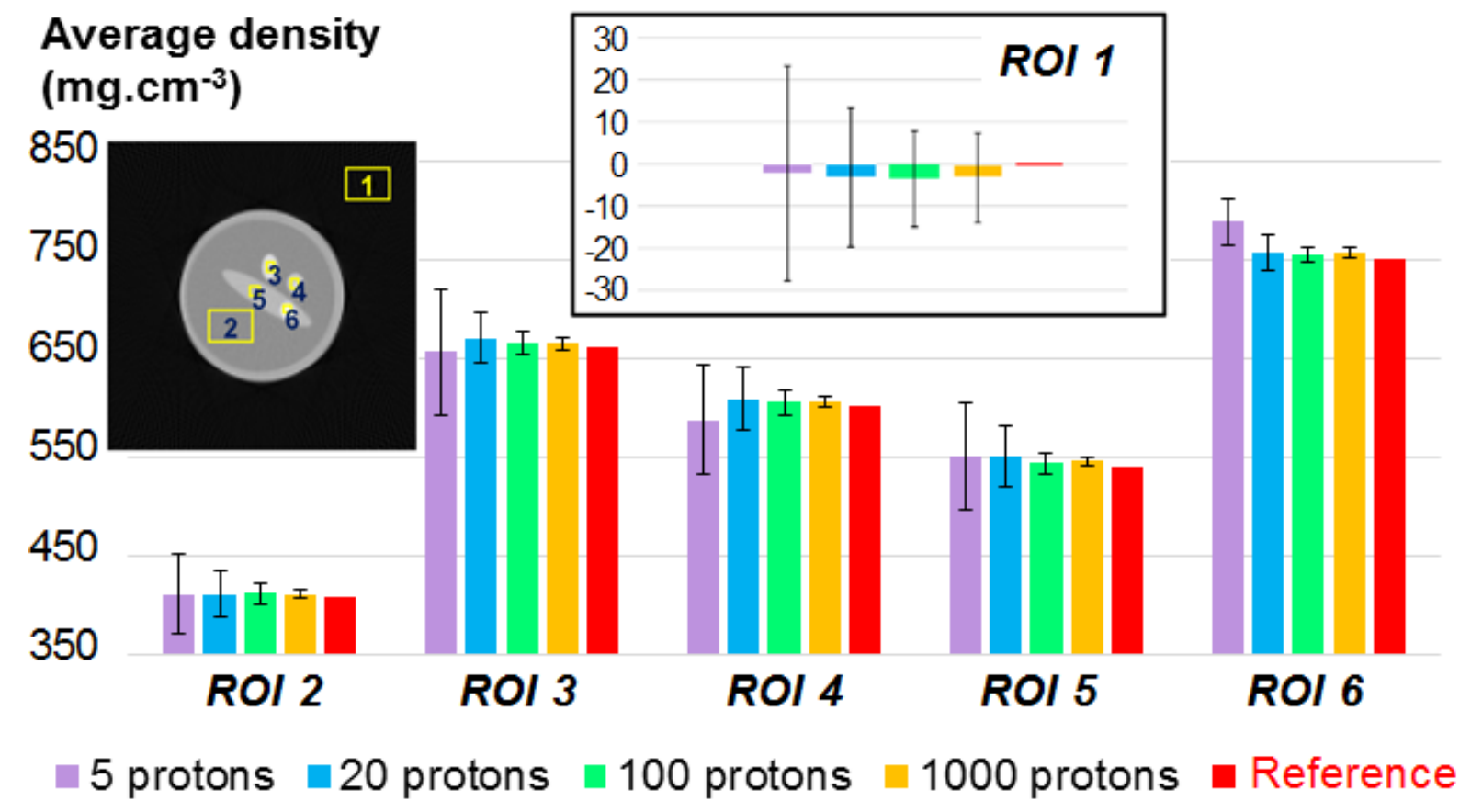


Fig.7

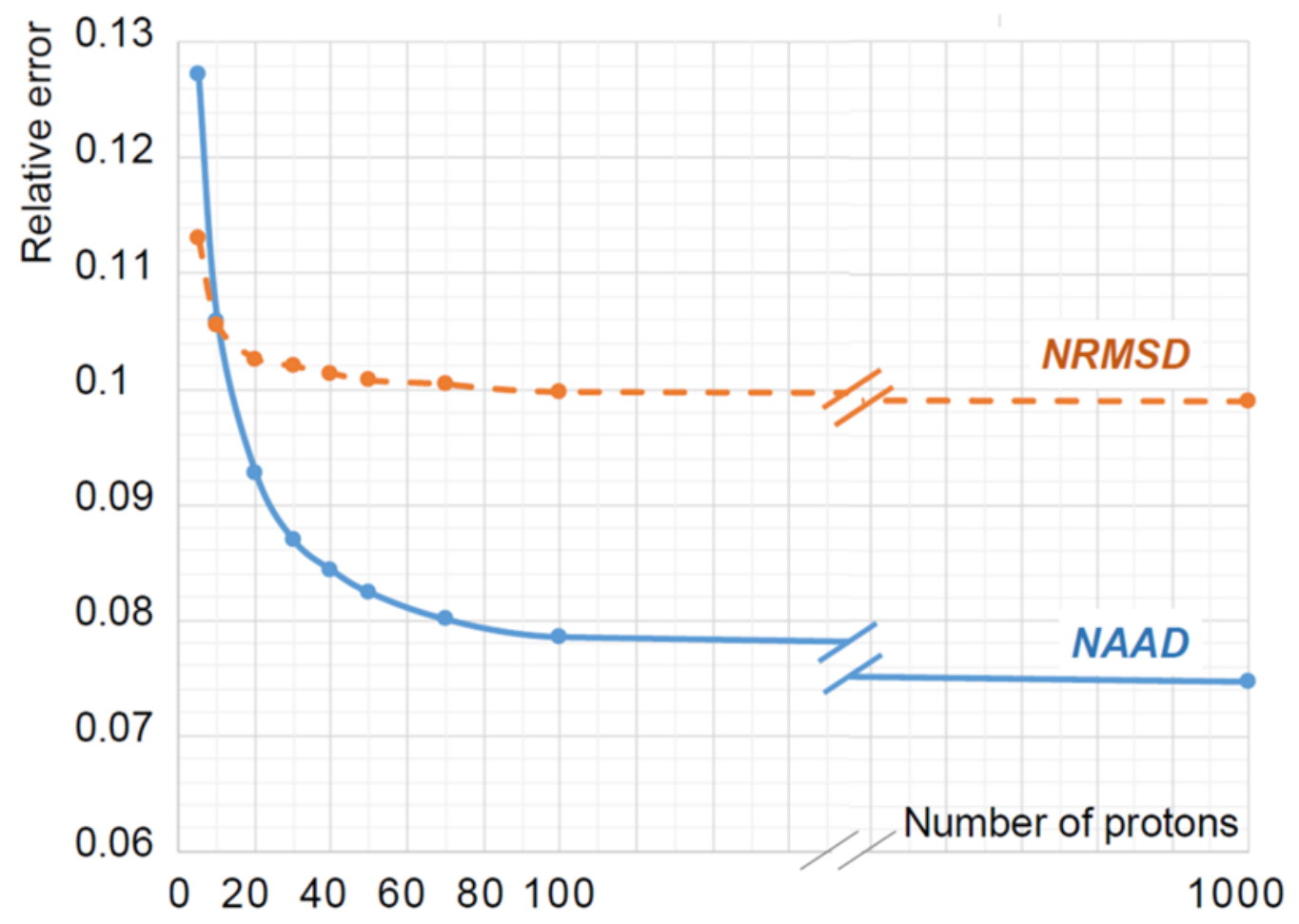


Fig.8

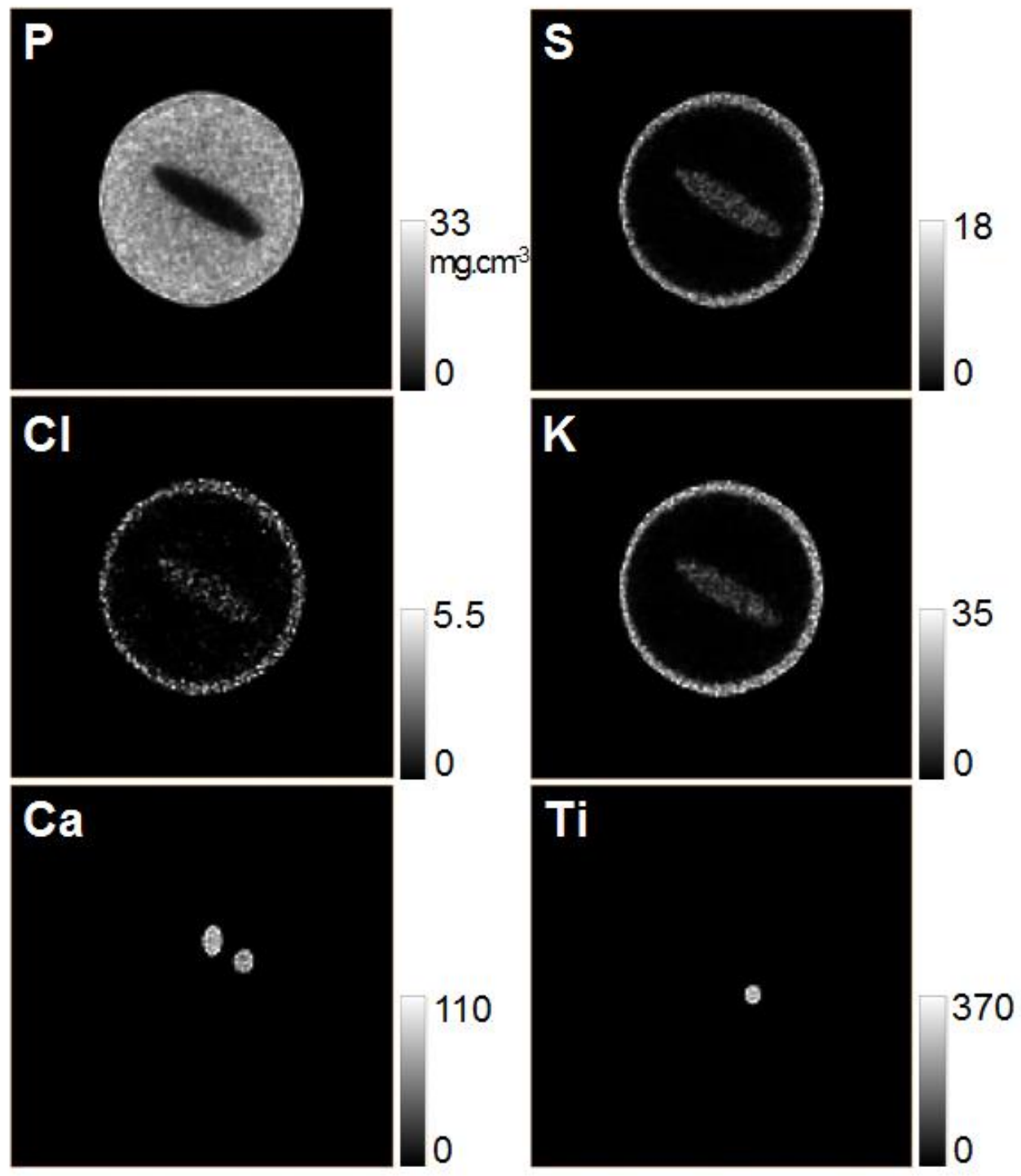


Fig.S1

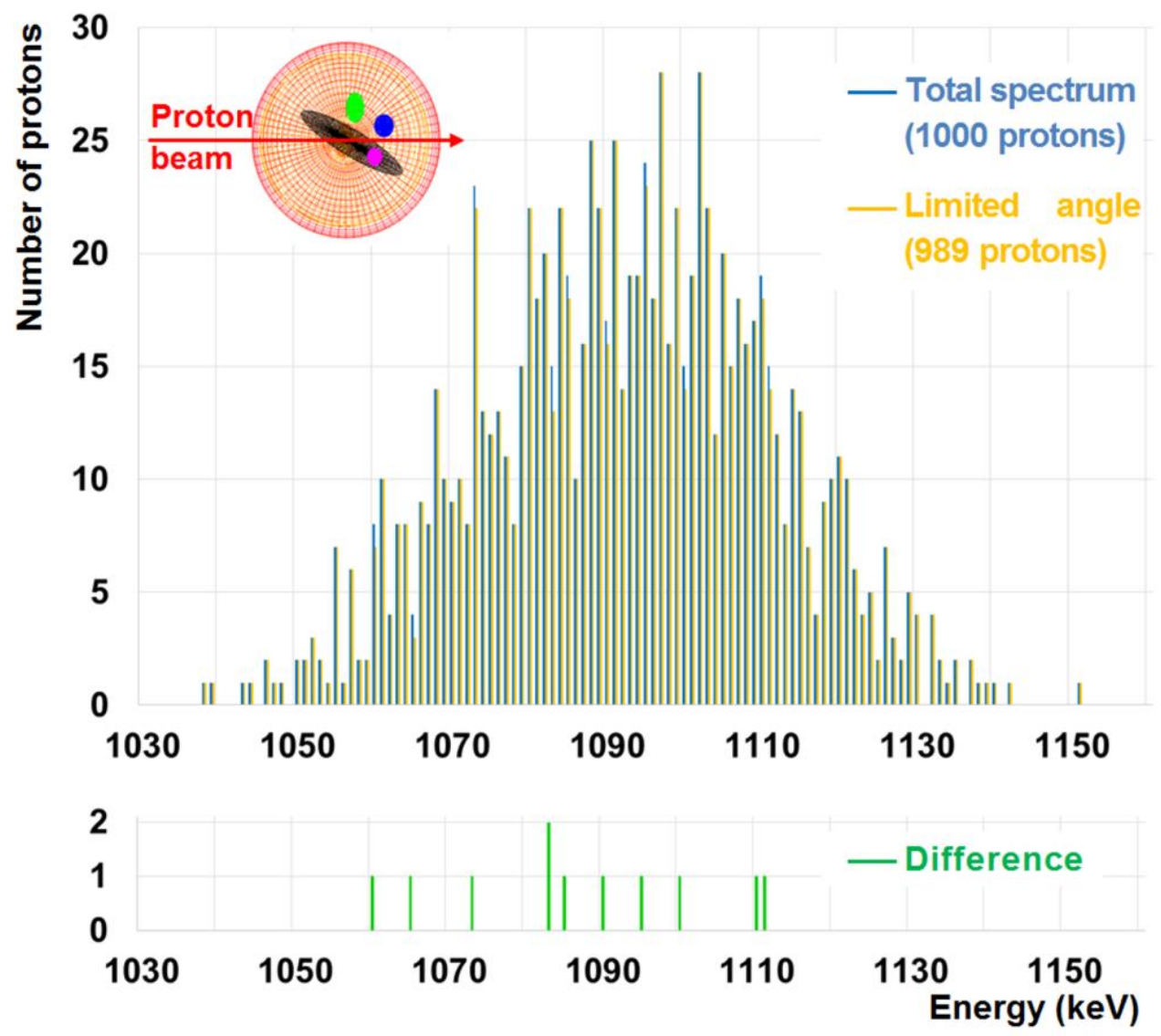


Fig. S2

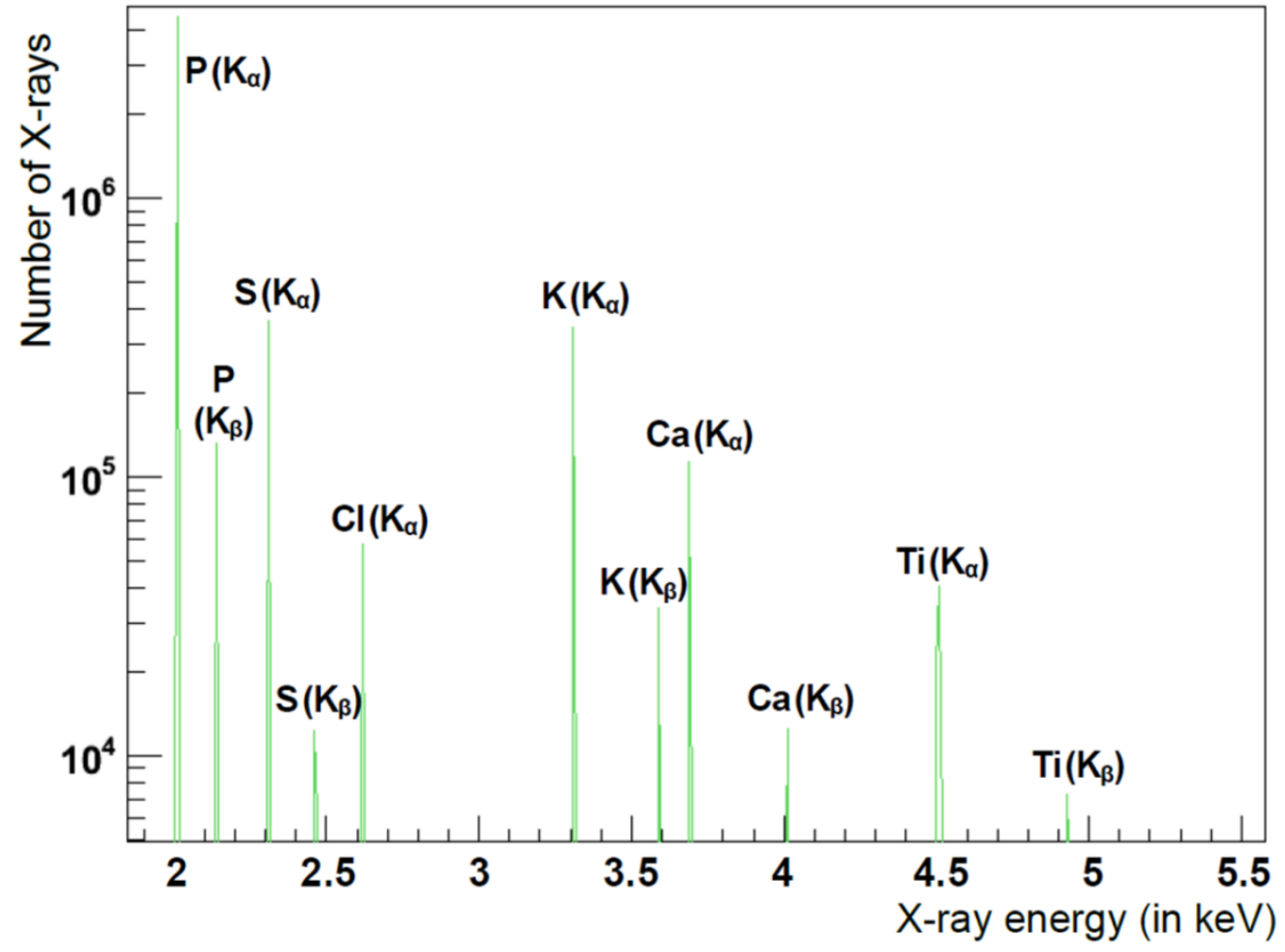


Fig. S3

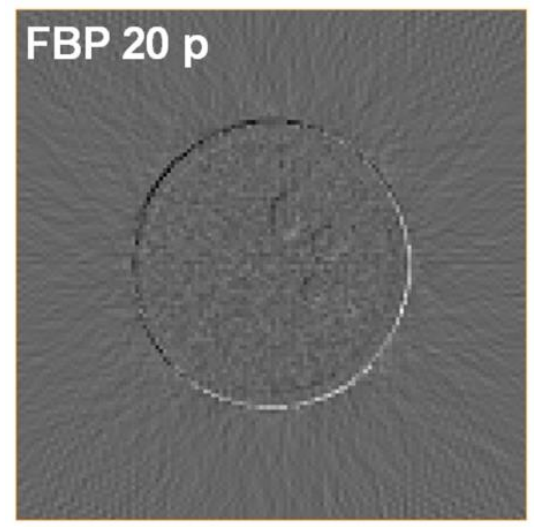

FBP $1000 p$
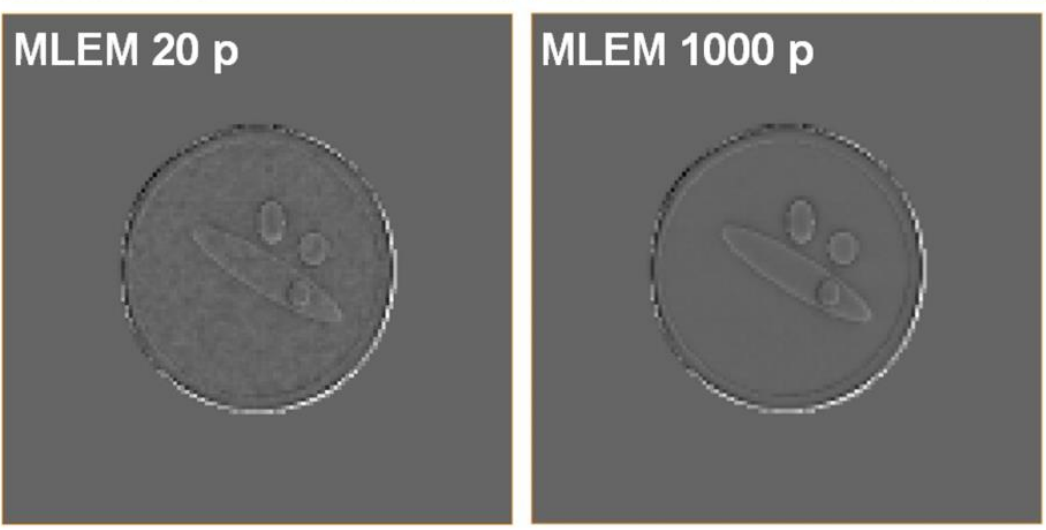
Fig. S4

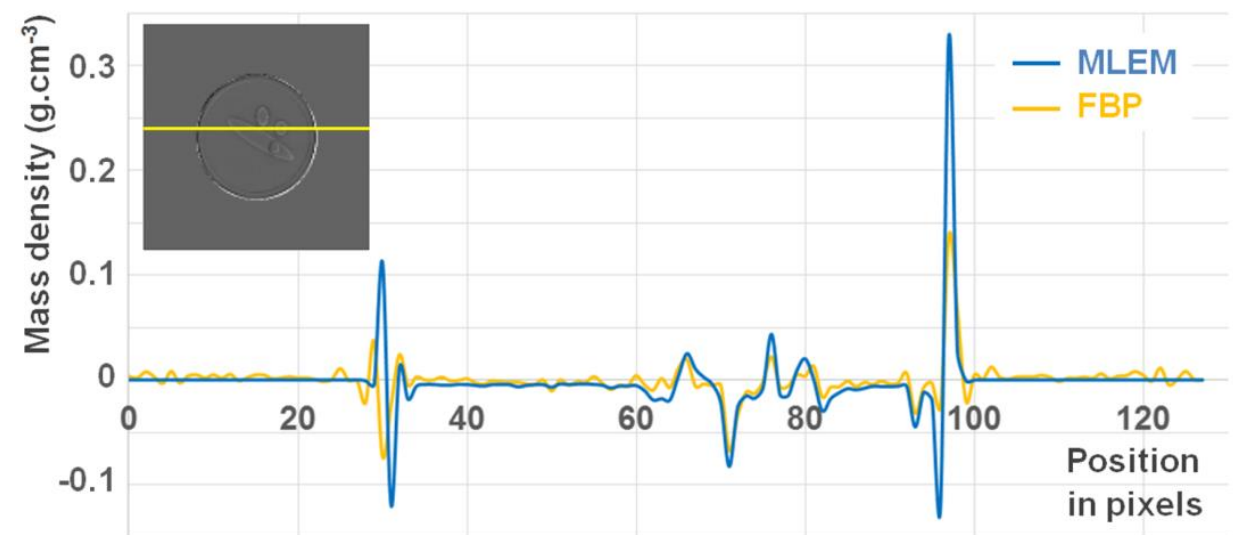

